\title{
Is shear wave elastography relevant in the diagnosis of polycystic ovarian syndrome?
}

\author{
Ersen Ertekin $^{1}$, Özgür Deniz Turan², Özüm Tunçyürek ${ }^{1}$
}

${ }^{1}$ Department of Radiology, ${ }^{2}$ Department of Obstetrics and Gynaecology Surgery, Aydin Adnan Menderes University Faculty of Medicine, Aydin, Turkey

\begin{abstract}
Aim: The aim of this study was to determine the contribution of Shear Wave Elastography (SWE) to the diagnosis of polycystic ovarian syndrome (PCOS). Material and methods: Thirty-seven patients with PCOS diagnosis criteria were included in the study. Sixteen volunteer patients without hormonal disturbances and with normal menstrual cycles were evaluated as the control group. Gray scale ultrasonography (US) and SWE measurements in both ovaries were performed by a single radiologist who was blinded to the clinical and laboratory results. Results: The SWE measurements in PCOS group were $8.4 \pm 2.0 \mathrm{kPa}$ for the right ovary and $9.4 \pm 3.9 \mathrm{kPa}$ for the left ovary and in the control group $7.8 \pm 4.1$ for the right ovary and $8.6 \pm 2.5 \mathrm{kPa}$ for the left ovary. There was no statistically significant difference between the PCOS and the control group according to the SWE results (for right ovary $\mathrm{p}=0.356$, for left ovary $\mathrm{p}=0.258$, and total ovary $\mathrm{p}=0.293$ ). Conclusions: The ovarian morphology is still the most reliable imaging finding in the diagnosis of PCOS, although it is controversial especially among adolescents. Although the diagnostic efficacy of SWE is demonstrated in a variety of soft tissue lesions, we did not find any significant contribution of SWE to the diagnosis PCOS. Therefore, the promising value of elastography is yet to be defined for the diagnosis of PCOS.
\end{abstract}

Keywords: polycystic ovary syndrome; ultrasonography; Shear Wave Elastography

\section{Introduction}

The need for a new and objective imaging finding that will contribute to the diagnosis of polycystic ovarian syndrome (PCOS) has been discussed as US emerges as a leading diagnostic tool [1]. The incidence of PCOS has increased over the decades and, as a complex genetic endocrine disorder, the increased number of women with PCOS must be aware of adverse health consequences of the disease [1-3].

Received 18.12.2018 Accepted 24.02.2019

Med Ultrason

2019, Vol. 21, No 2, 158-162

Corresponding author: Ersen Ertekin, MD

Department of Radiology

Aydin Adnan Menderes University

Faculty of Medicine, Aydin, Turkey

Phone: +90 (256) 444-1256

Fax: +90 (256) 214-6495

E-mail: drersen@hotmail.com
The diagnosis usually deals with the clinical presentation of the PCOS as a menstrual disorder, obesity and hirsutism, infertility, diabetes mellitus and a variety of co-existing metabolic syndromes $[4,5]$. Therefore, evidence-based management of PCOS allows the reduction of metabolic complications in patients with this distinct diagnosis.

The current diagnostic criteria for the diagnosis of PCOS, which are named as the "Rotterdam criteria" consists of at least two of the following clinical settings: oligo/ anovulation, hyperandrogenism or polycystic ovarian morphology (PCOM) [6]. However, these criteria are particularly controversial for the diagnosis of PCOS in adolescents [7]. In ultrasound, polycystic ovarian appearance can be mistaken for other diseases or even misdiagnosed in healthy subjects [8,9].

We speculated that the need for an additional quantifiable parameter is mandatory to for an accurate PCOS diagnosis. Therefore, we implemented ultrasound (US) elastography (USE) for ovarian screening, as elastog- 
raphy evidently provides reliable parameters to differentiate solid lesions from fibrotic/cystic tissue [10-16]. Moreover, data obtained by USE supplies complementary information to gray scale US by adding stiffness as a measurable property to current US technique [16].

In general, the USE technique can be grouped under two headings: 1) strain elastography (SE) - a semiquantitative technique that shows the resistance of the tissue to deformation against a mechanical stress by means of Young's modulus and 2) shear wave elastography (SWE) that provides quantitative data by measuring the speed of the shear waves created by mechanical vibration device or acoustic radiation force. Shear wave velocity (meters $/$ second $=\mathrm{m} / \mathrm{s}$ ) can be converted to pressure unit (kilopascal=kPa) by Young's modulus. SWE has lower operator dependency and higher reliability compared to strain elastography [16].

The aim of the present study was to determine the contribution of SWE for the diagnosis of PCOS.

\section{Material and methods}

Approval was obtained from the local Ethics Committee for this study. A signed voluntary information consent form was obtained from all the participants in the study.

One hundred and fifty-six female patients admitted to the infertility outpatient department between January 2017 and January 2018 were evaluated according to the Rotterdam criteria for PCOS diagnosis. Thirty-seven patients who matched these criteria were included in the study. Sixteen volunteer patients with no hormonal imbalance and normal menstrual cycles were evaluated as control group.

For all patients, gray scale US (ovarian size, presence, number and size of cysts), SWE measurements and laboratory tests (FSH, LH, Progesterone, DHEA) were performed on the 3rd day of the menstrual cycle. Gray scale
US and SWE measurements were performed by a single radiologist who was blinded to the clinical and laboratory results. In gray scale US, ovaries were evaluated in terms of PCOM. The criteria for PCOM were based on the presence of at least 12 cysts $<10 \mathrm{~mm}$ per ovary and/or $>10 \mathrm{ml}$ of ovarian volume. The cases interpreted as suspicious for PCOM were re-evaluated with the participation of a second radiologist and the final decisions were made by consensus.

All US and SWE measurements were performed transabdominally on a Samsung RS80 US device (Gyeonggi-do, Republic of Korea) using a $6 \mathrm{MHz}$ convex probe by a radiologist with 6 years of experience in USE. First, the number of cysts was evaluated and the ovarian volumes were measured by gray scale US. Then, in SWE evaluation, cysts were excluded from the measurement area and all SWE measurements were performed from the ovarian stroma, at least 10 consecutive measurements for each ovary. For the optimization of the SWE measurements, the quality factor (RMI), which the device automatically provides, was considered to be between 0.4-1.0. The RMI values below 0.4 were excluded. The average of 10 measurements with the appropriate quality factor was recorded as the elasticity of that ovary. The unit of SWE measurement was determined as kilopascal (kPa).

\section{Statistical analysis}

Statistical analysis was performed by SPSS 22.0. The differences between the groups were evaluated with the Mann-Whitney U test. Kolmogrov-Simirnov normality test was used to determine the distribution of data. Because the data were not distributed normally, correlation analyses were evaluated with non-parametric Spearman's Correlation test. ROC (receiver operating characteristic) analysis was performed in order to determine the threshold value for the ovarian volumes which has significant correlation with the diagnosis of PCOS. A p value of $<0.05$ was considered statistically significant.

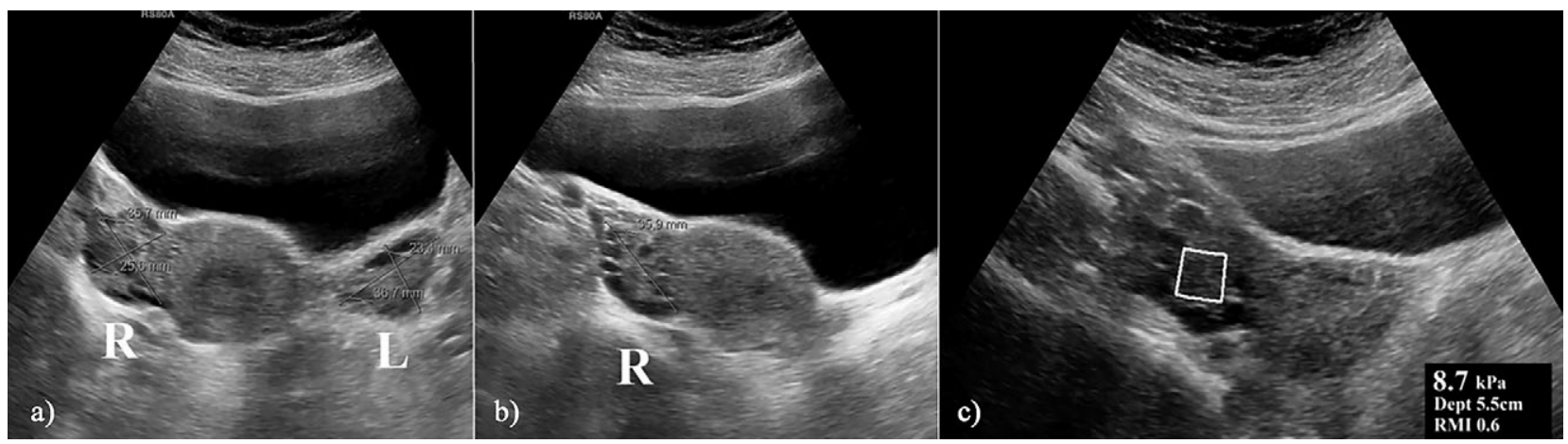

Fig 1. The gray scale ( $a$ and b) and shear wave elastography (c) measurements of the right ovary in PCOS patient. 


\section{Results}

The demographic data and ultrasound measurement (gray scale and SWE) are detailed in Table I. Figures 1 and 2 show the modality of measurements in gray scale and SWE in a PCOS patient and control, respectively.

The sensitivity and specificity of ovarian volume for PCOS diagnosis were $82 \%$ and $82 \%$, respectively, when $7 \mathrm{ml}$ was considered as cut-off value. If $7.5 \mathrm{ml}$ was accepted as the cut-off value, the sensitivity and specificity values were $80 \%$ and $94 \%$ respectively.

In Spearman's correlation analysis, no correlations between the elasticity of the ovarian stroma and age, height, weight, BMI and the ovarian volumes were found $(r=0.140, p=0.347 ; r=0.155, p=0.368 ; r=0.005, p=0.975$; $\mathrm{r}=0.156, \mathrm{p}=0.174 ; \mathrm{r}=0.181, \mathrm{p}=0.145$, respectively).

\section{Discussion}

In our study, there was no correlation between SWE results and conventional parameters used in diagnosis of PCOS. Although a slight correlation of SWE with BMI and ovarian volume was detected, clinical relevance of SWE to determine PCOS was not obvious given the results of our study. Our results are in contrast with Ciraci et al study [17], the authors demonstrating high stiffness of the ovarian stroma when PCOS is present. However, Ciraci et al [17] used SE which is highly subjective and lacks quantifiable parametric values. We used SWE to evaluate elasticity of ovarian stroma, a method in which the elasticity is objectively calculated by the regular software of US device [15]. Also, our patients were younger than those in the Ciraci et al [17] study (21.4 \pm 3.7 and $25.7 \pm 4.2$, respectively). In addition, the difference may be caused by the lack of superpose infections and/or drug interactions that may be the cause of fibrosis, due to the shorter disease process. Therefore, we conclude that SWE does not bring additional data for the diagnosis of
PCOS and conventional US diagnostic criteria are still valid.

As is known, PCOS, which is one of the causes of significant infertility in reproductive women, is a syndrome with oligo/amenorrhea. For the diagnosis, the use of Rotterdam criteria consisting of oligo/anovulation, hyperandrogenism and PCOM is generally accepted [6]. Diagnostic potential for PCOS was highest for the follicle number (0.969) and the ovarian volume (0.873) as judged by the area under the ROC curve. A follicle number threshold of 26 follicles had the best compromise between sensitivity (85\%) and specificity (94\%) when discriminating between controls and PCOS. The ovarian volume of $10 \mathrm{ml}$ had $81 \%$ sensitivity and $84 \%$ specificity [18]. But these values are low for the diagnosis of PCOS. However, PCOM is controversial, especially in adolescents. Although there are publications reporting an increase in the total and stromal ovarian volume with an increase in the number of cysts [19-23], there are also publications indicating that there is no significant increase in the ovarian volume [24,25]. Furthermore, Alsamarai et al [26] concluded that ovarian volume (OV) and follicle number decrease with age in women with PCOS. Controversially, Christensen et al [27] found that OV was not related to age in 428 healthy women aged between 18 and 45 years. In addition, there are also reports that the ovarian volume and the numbers of cysts may be different in different ethnic communities [28-30]. The threshold for distinguishing polycystic ovaries from normal ovaries varied from 8 to $17.6 \mathrm{ml}$ among different studies. Kosus et al [30] found that $6.43 \mathrm{ml}$ was a diagnostic threshold of OV for Turkish women with PCOS, which is a lower diagnostic threshold in comparison to the Rotterdam criteria and this indicates ethnicity variations. In our study, we found lower ovarian volume values compared to Rotterdam criteria. In accordance with literature, ovarian volumes were significantly higher in the PCOS group compared to the control group. When a cut-off value of

Table I. Summary of the demographic data and ultrasonographic measurements in patient and control groups

\begin{tabular}{lllll}
\hline & & PCOS group $(\mathbf{n}=\mathbf{3 7})$ & Control group $(\mathbf{n}=\mathbf{1 6})$ & $\mathbf{p}$ value \\
\hline Age $($ year) & & $21.5 \pm 3.7$ & $23.0 \pm 5.0$ & 0.293 \\
$\mathrm{BMI}\left(\mathrm{kg} / \mathrm{m}^{2}\right)$ & $24.5 \pm 4.8$ & $25.5 \pm 3.8$ & 0.698 \\
Oligo/amenore & $37(100)$ & $0(0)$ & $<0.001$ \\
Elevation of DHEA-SO & 4 & NA & $<0.001$ \\
Policystic appearance & & $34(92)$ & $5(31)$ & 0.356 \\
SWE $(\mathrm{kPa})$ & Right ovary & $80(81)$ & $7.8 \pm 4.1$ & 0.258 \\
& Left ovary & $9.4 \pm 3.9$ & $8.6 \pm 2.5$ & 0.001 \\
Ovary volume $(\mathrm{ml})$ & Right & $11.3 \pm 4.6$ & $5.4 \pm 1.8$ & 0.001 \\
\hline
\end{tabular}

The results are expressed as a mean \pm standard deviation or number (\%). NA: Not available, PCOS: Polycystic ovarian syndrome, BMI: Body Mass Index, DHEA-SO4: dehidroepiandrosteron, SWE: Shear wave elastography 


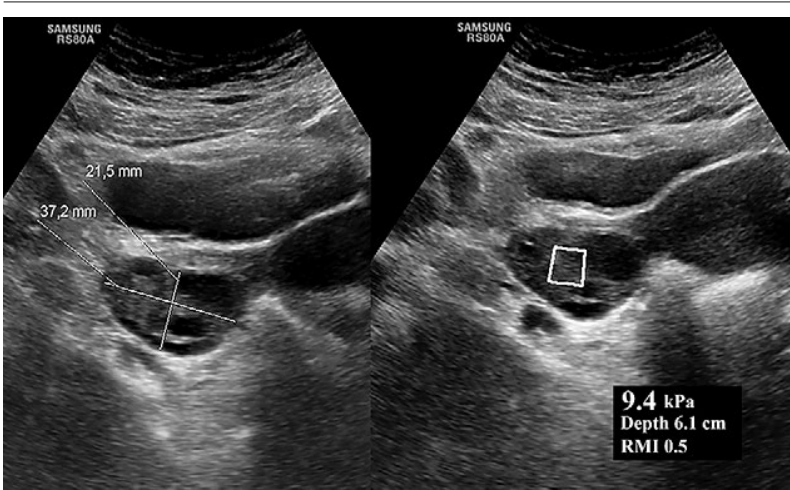

Fig 2. The gray scale (a) and shear wave elastography (b) measurements of the right ovary in control.

$7.5 \mathrm{ml}$ was accepted for the ovarian volume, we obtained $80 \%$ sensitivity and $94 \%$ specificity.

In addition to factors such as age and ethnicity which may cause changes in ovarian volume, PCOM appearance and volume increase in women with completely normal physiology have led to the search of new diagnostic criteria for PCOS [8,27,31]. Although new diagnostic criteria, such as increasing the number of cysts from 12 to 25, the use of stromal volume / total ovarian volume ratio and Doppler US were studied, none of them have gained general acceptance [18,32-36]. Therefore, the establishment of more objective diagnostic criteria for the diagnosis of PCOS remains important.

In recent studies, it has been envisaged that the stroma of the ovaries with PCOS is expected to be stiffened due to the increase of teca cells and the thickened fibrotic albuginea. In animal experiments, the presence of fibrosis in ovarian tissue was histopathologically revealed [3741]. In humans, fibrosis in the ovarian tissue can be demonstrated non-invasively with elastography [10-13]. This current study contributes to the literature in this respect.

Our study has some limitations. First, during SWE measurement, small cysts may have entered the measurement area, although we tried not to take the cysts into the ROI and this may have reduced the stromal elasticity. In our USE system ROI was $2 \mathrm{~mm}^{2}$. The second limitation may be that the patient group was a young group. Since the young patients were in the early stages of the PCOS process, histopathological changes had not occurred yet. An larger group concerning the age of PCOS patients would probably offer different results. However, we preferred to analyze a younger patient group because the criteria of Rotterdam are more controversial in adolescents. The third limitation was the lack of an interoberver/intraobserver agreement.

In conclusion although the diagnostic efficacy of USE has been proven in many organs and pathologies, we did not find any significant difference in the ovarian stromal stiffness in patients with PCOS compared with normal patients. Further studies are required with larger patient populations and a larger age interval.

\section{Conflict of interest: None}

Acknowledgement: thanks to Jennifer Ozturkeri for language editing of the manuscript.

\section{References}

1. Ganie MA, Kalra S. Polycystic ovary syndrome - A metabolic malady, the mother of all lifestyle disorders in women - Can Indian health budget tackle it in future? Indian J Endocrinol Metab 2011;15:239-241.

2. Khan KA, Stas S, Kurukulasuriya LR. Polycystic ovarian syndrome. J Cardiometab Syndr 2006;1:125-130.

3. Ibanez L, Oberfield SE, Witchel S, et al. An International Consortium Update: Pathophysiology, Diagnosis, and Treatment of Polycystic Ovarian Syndrome in Adolescence. Horm Res Paediatr 2017;88:371-395.

4. Orsino A, Van Eyk N, Hamilton J. Clinical features, investigations and management of adolescents with polycystic ovary syndrome. Paediatr Child Health 2005; 10:602-608.

5. Cunningham P. Pathophysiology, diagnosis and treatment of polycystic ovary syndrome. Nurs Stand 2017;31:4451.

6. Goodman NF, Cobin RH, Futterweit W, et al. American Association of Clinical Endocrinologists, American College of Endocrinology, and Androgen Excess and PCOS Society Disease State Clinical Review: Guide to the Best Practices in the Evaluation and Treatment of Polycystic Ovary Syndrome - Part 2. Endocr Pract 2015;21:1415-1426.

7. Rackow BW. Polycystic ovary syndrome in adolescents. Curr Opin Obstet Gynecol 2012;24:281-287.

8. Senaldi L, Gopi RP, Milla S, Shah B. Is ultrasound useful in the diagnosis of adolescents with polycystic ovary syndrome? J Pediatr Endocrinol Metab 2015;28:605-612.

9. Dewailly D. Ultrasound definition of polycystic ovarian morphology: good news and bad news. Fertil Steril 2014;101:49-50.

10. Rattansingh A, Amooshahi H, Menezes RJ, et al. Utility of shear-wave elastography to differentiate low from advanced degrees of liver fibrosis in patients with hepatitis $\mathrm{C}$ virus infection of native and transplant livers. J Clin Ultrasound 2018;46:311-318.

11. Chen SH, Lai HC, Chiang IP, et al. Changes in liver stiffness measurement using acoustic radiation force impulse elastography after antiviral therapy in patients with chronic hepatitis C. PLoS One 2018;13:e0190455.

12. Lee MS, Bae JM, Joo SK, et al. Prospective comparison among transient elastography, supersonic shear imaging, and ARFI imaging for predicting fibrosis in nonalcoholic fatty liver disease. PLoS One 2017;12:e0188321.

13. Ahn SJ, Lee JM, Chang W, et al. Prospective Validation of Intra- and Interobserver Reproducibility of a New Point 
Shear Wave Elastographic Technique for Assessing Liver Stiffness in Patients with Chronic Liver Disease. Korean J Radiol 2017;18:926-935.

14. Dewall RJ. Ultrasound elastography: principles, techniques, and clinical applications. Crit Rev Biomed Eng 2013;41:1-19.

15. Ooi CC, Malliaras P, Schneider ME, Connell DA. "Soft, hard, or just right?" Applications and limitations of axialstrain sonoelastography and shear-wave elastography in the assessment of tendon injuries. Skeletal Radiol 2014;43:112 .

16. Sigrist RMS, Liau J, Kaffas AE, Chammas MC, Willmann JK. Ultrasound Elastography: Review of Techniques and Clinical Applications. Theranostics 2017;7:1303-1329.

17. Ciraci S, Tan S, Ozcan AS, et al. Contribution of real-time elastography in diagnosis of polycystic ovary syndrome. Diagn Interv Radiol 2015;21:118-122.

18. Lujan ME, Jarrett BY, Brooks ED, et al. Updated ultrasound criteria for polycystic ovary syndrome: reliable thresholds for elevated follicle population and ovarian volume. Hum Reprod 2013;28:1361-1368.

19. Balen AH. Ovulation induction in the management of anovulatory polycystic ovary syndrome. Mol Cell Endocrinol 2013;373:77-82.

20. Lee TT, Rausch ME. Polycystic ovarian syndrome: role of imaging in diagnosis. Radiographics 2012;32:1643-1657.

21. Zhu RY, Wong YC, Yong EL. Sonographic evaluation of polycystic ovaries. Best Pract Res Clin Obstet Gynaecol 2016;37:25-37.

22. Lam P, Raine-Fenning N, Cheung L, Haines C: Threedimensional ultrasound features of the polycystic ovary in Chinese women. Ultrasound Obstet Gynecol 2009;34:196200.

23. Han YS, Lee AR, Song HK, et al. Ovarian Volume in Korean Women with Polycystic Ovary Syndrome and Its Related Factors. J Menopausal Med 2017;23:25-31.

24. Ybarra M, Franco RR, Cominato L, Sampaio RB, Sucena da Rocha SM, Damiani D. Polycystic Ovary Syndrome among Obese Adolescents. Gynecol Endocrinol 2018;34:45-48.

25. Hickey M, Doherty DA, Atkinson H, et al. Clinical, ultrasound and biochemical features of polycystic ovary syndrome in adolescents: implications for diagnosis. Hum Reprod 2011;26:1469-1477.

26. Alsamarai S, Adams JM, Murphy MK, et al. Criteria for polycystic ovarian morphology in polycystic ovary syndrome as a function of age. J Clin Endocrinol Metab 2009;94:4961-4970.

27. Christensen JT, Boldsen J, Westergaard JG. Ovarian volume in gynecologically healthy women using no contraception, or using IUD or oral contraception. Acta Obstet Gynecol Scand 1997;76:784-789.

28. Atiomo WU, Pearson S, Shaw S, Prentice A, Dubbins P. Ultrasound criteria in the diagnosis of polycystic ovary syndrome (PCOS). Ultrasound Med Biol 2000;26:977980.

29. Chen Y, Li L, Chen X, et al. Ovarian volume and follicle number in the diagnosis of polycystic ovary syndrome in Chinese women. Ultrasound Obstet Gynecol 2008;32:700703 .

30. Kosus N, Kosus A, Turhan NO, Kamalak Z. Do threshold values of ovarian volume and follicle number for diagnosing polycystic ovarian syndrome in Turkish women differ from western countries? Eur J Obstet Gynecol Reprod Biol 2011; 154:177-181.

31. Dewailly D, Lujan ME, Carmina E, et al. Definition and significance of polycystic ovarian morphology: a task force report from the Androgen Excess and Polycystic Ovary Syndrome Society. Hum Reprod Update 2014;20:334-352.

32. Battaglia C, Battaglia B, Morotti E, et al. Two- and threedimensional sonographic and color Doppler techniques for diagnosis of polycystic ovary syndrome. The stromal/ovarian volume ratio as a new diagnostic criterion. J Ultrasound Med 2012;31:1015-1024.

33. Makled AK, El Sherbiny M, Elkabarity R. Assessment of ovarian stromal blood flow after metformin treatment in women with polycystic ovary syndrome. Arch Gynecol Obstet 2014;289:883-891.

34. Lie Fong S, Laven JSE, Duhamel A, Dewailly D. Polycystic ovarian morphology and the diagnosis of polycystic ovary syndrome: redefining threshold levels for follicle count and serum anti-Mullerian hormone using cluster analysis. Hum Reprod 2017;32:1723-1731.

35. Wongwananuruk T, Panichyawat N, Indhavivadhana S, et al. Accuracy of anti-Mullerian hormone and total follicles count to diagnose polycystic ovary syndrome in reproductive women. Taiwan J Obstet Gynecol 2018;57:499-506.

36. Sujata K, Swoyam S. 2D and 3D Trans-vaginal Sonography to Determine Cut-offs for Ovarian Volume and Follicle Number per Ovary for Diagnosis of Polycystic Ovary Syndrome in Indian Women. J Reprod Infertil 2018;19:146151.

37. Zhou F, Shi LB, Zhang SY. Ovarian Fibrosis: A Phenomenon of Concern. Chin Med J (Engl) 2017;130:365-371.

38. Miao ZL, Guo L, Wang YX, et al. The intervention effect of Rosiglitozone in ovarian fibrosis of PCOS rats. Biomed Environ Sci 2012;25:46-52.

39. Zhang X, Zhang C, Shen S, et al. Dehydroepiandrosterone induces ovarian and uterine hyperfibrosis in female rats. Hum Reprod 2013;28:3074-3085.

40. Takahashi N, Harada M, Hirota Y, et al. Activation of Endoplasmic Reticulum Stress in Granulosa Cells from Patients with Polycystic Ovary Syndrome Contributes to Ovarian Fibrosis. Sci Rep 2017;7:10824.

41. Wang D, Wang W, Liang Q, et al. DHEA-induced ovarian hyperfibrosis is mediated by TGF-beta signaling pathway. J Ovarian Res 2018;11:6. 